\title{
Oxidation of Thin Al Layers for Magnetic Tunnel Barriers: a Three-dimensional Atom Probe Study.
}

\author{
A. K. Petford-Long, ${ }^{*}$ D.J. Larson, ${ }^{* *}$ A. Cerezo, ${ }^{* * *}$ P.H. Clifton, ${ }^{* * * *}$ S. Pinitsoontorn*** \\ * Materials Science Division, Argonne National Lab, 9700 S Cass Ave, Argonne, IL 60439 \\ **Seagate Technology, 1 Disc Drive, Bloomington, MN 55435. \\ *** Dept of Materials, University of Oxford, Oxford OX1 3PH, UK \\ ****Oxford Nanoscience, 4 Carters Lane, Milton Keynes MK11 3ER, UK
}

Magnetic tunnel junction structures are of interest because of the tunnelling magnetoresistance (TMR) that they display [1], and because of their potential applications as magnetic sensors and in magnetic random access memory arrays. The TMR phenomenon relies on spin-dependent tunnelling across a nanoscale oxide tunnel barrier, with the morphology and composition of the barrier, and of the interfaces between it and the adjacent ferromagnetic layers playing a critical role in controlling the tunnelling. The material most widely used for the tunnel barrier is aluminium oxide, formed by depositing $\mathrm{Al}$ on the underlying ferromagnetic layer, followed by oxidation to form an barrier. The process by which oxidation occurs is not easy to characterise because the final tunnel barrier layer is only $\sim 1 \mathrm{~nm}$ thick and is encapsulated between two magnetic layers.

We have followed the process by which a continuous tunnel barrier is formed in a simple magnetic tunnel junction structure consisting of $\mathrm{Si} / \mathrm{seed} / \mathrm{CoFe}_{6 \mathrm{~nm}} / \mathrm{Al}_{0.6 \mathrm{~nm}}$ (oxidised) $/ \mathrm{CoFe}_{3 \mathrm{~nm}} /$ cap. The degree of oxidation was changed by increasing oxidation time from $0.1 t_{o x}$ to $t_{o x}$, where $t_{o x}$ is the time required for full oxidation of the Al. The effect of annealing on the final structure was also studied as this is known to improve the transport and magnetic properties of the barriers. For a highly underoxidized condition $\left(0.1 \mathrm{t}_{\mathrm{ox}}\right.$, Fig. 1a) the tunnel barrier consists of Al-oxide rich islands distributed across the nominal barrier plane. The HREM image shows only partial formation of the pale contrast amorphous oxide layer across the image. The reconstructed 3DAP data are displayed as white surfaces enclosing regions in which $\mathrm{Al}+\mathrm{O}$ is greater than 15 at $\%$, and as a thermal slice indicating the $\mathrm{Al}+\mathrm{O}$ composition across a $0.4 \mathrm{~nm}$ thick layer in the barrier plane. Even within the oxide islands there is still a high concentration of $\mathrm{Co}$ and the $\mathrm{Al}: \mathrm{O}$ ratio is $2: 1$. Balling up of a very thin Al layer following oxidation has been predicted using molecular dynamics simulations [2] and our data agree well with these predictions. When the oxidation time is increased to $0.25 \mathrm{t}_{\mathrm{ox}}$ (Fig. 1b) the oxide islands increase in size but are still well isolated, and even for full oxidation, i.e. 1.0t $\mathrm{t}_{\mathrm{ox}}$ (Fig. 1c) the oxide is still not continuous but now forms a network with a length scale of the same order as the grain size, suggesting that the oxide is forming at the positions of grain boundaries in the underlying $\mathrm{CoFe}$ layer. It is only after annealing at $250^{\circ} \mathrm{C}$ (Fig. 2) that a continuous oxide barrier can be seen in the HREM image, and the composition profile now shows an $\mathrm{Al}$ :O ratio in the barrier of 2:3 as would be expected for stoichiometric alumina.

At first sight it might appear unfavorable for annealing to produce a continuous thin film oxide layer but there are a number of factors that must be considered. Firstly, the two CoFe layers do not grow epitaxially on one another: between the oxide islands small amounts of $\mathrm{Al}$ and $\mathrm{O}$ lead to the presence of a grain boundary at the original surface. Secondly the closure of small holes due to the energy of the edge of the hole has been shown theoretically to be favorable [3]. However the largest effect is that of strain introduced during annealing because of the increased oxygen content of the alumina. For the Co-Al-O system being considered, it can be shown that it is favorable for the oxide to adopt a platelet-like morphology. For realistic values of the metal/oxide interface energy $\left(1 \mathrm{~J} \mathrm{~m}^{-2}\right)$ 
(lower than would be expected for pure alumina because of the high Co content) the oxide can in fact spread to form a continuous layer as has been observed.

[1] E. Y. Tsymbal, O. N. Mryasov, P. R. LeClair, J. Phys. Cond. Matt. 15 (2003) R109.

[2] X. Zhou and H.G.N. Wadley, Phys. Rev. B71 (2005) 054418.

[3]X. Zhou and H.G.N. Wadley, Phys. Rev. B (in Press, Feb 2006).
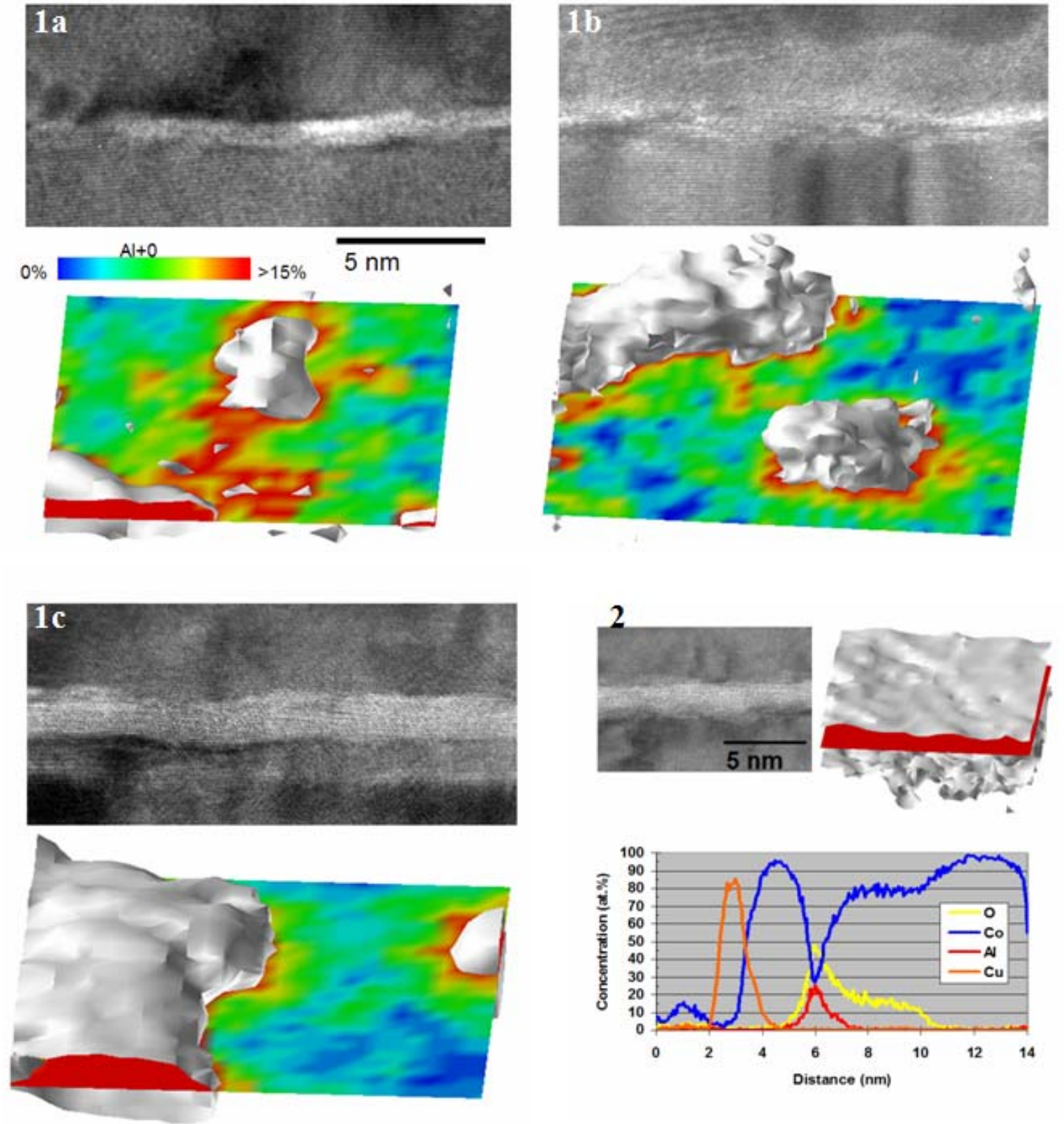

Fig. 1. HREM images and atom probe data displayed as a thermal slice through the plane containing the highest oxide content and isoconcentration surfaces enclosing the volume of composition of $\mathrm{Al}+\mathrm{O}>15$ at.\% for oxidation times of a) $0.1 \mathrm{t}_{\mathrm{ox}}$, b) $0.25 \mathrm{t}_{\mathrm{ox}}$ and c) $1.0 \mathrm{t}_{\mathrm{ox}}$.

Fig. 2. HREM image, isoconcentration surface and composition profile normal to barrier plane for annealed sample with oxidation time of $1.0 \mathrm{t}_{\mathrm{ox}}$. 